\title{
Experimental evidence for strong stabilizing forces at high functional diversity of aquatic microbial communities
}

\author{
Francesco Carrara, ${ }^{1,2,6}$ Andrea Giometto, ${ }^{1,3}$ Mathew Seymour, ${ }^{3}$ Andrea Rinaldo,,${ }^{1,4}$ \\ AND Florian Altermatt ${ }^{3,5}$ \\ ${ }^{1}$ Laboratory of Ecohydrology, École Polytechnique Fédérale Lausanne (EPFL), 1015 Lausanne, Switzerland \\ ${ }^{2}$ Ralph M. Parsons Laboratory, Department of Civil and Environmental Engineering, Massachusetts Institute of Technology, \\ Cambridge, Massachusetts 02139 USA \\ ${ }^{3}$ Department of Aquatic Ecology, Eawag: Swiss Federal Institute of Aquatic Science and Technology, 8600 Dübendorf, Switzerland \\ ${ }^{4}$ Dipartimento ICEA, Università di Padova, 35131 Padova, Italy \\ ${ }^{5}$ Institute of Evolutionary Biology and Environmental Studies, University of Zurich, 8057 Zürich, Switzerland
}

\begin{abstract}
Unveiling the mechanisms that promote coexistence in biological communities is a fundamental problem in ecology. Stable coexistence of many species is commonly observed in natural communities. Most of these natural communities, however, are composed of species from multiple trophic and functional groups, while theory and experiments on coexistence have been focusing on functionally similar species. Here, we investigated how functional diversity affects the stability of species coexistence and productivity in multispecies communities by characterizing experimentally all pairwise species interactions in a pool of 11 species of eukaryotes (10 protists and one rotifer) belonging to three different functional groups. Species within the same functional group showed stronger competitive interactions compared to among-functional group interactions. This often led to competitive exclusion between species that had higher functional relatedness, but only at low levels of species richness. Communities with higher functional diversity resulted in increased species coexistence and community biomass production. Our experimental findings and the results of a stochastic model tailored to the experimental interaction matrix suggest the emergence of strong stabilizing forces when species from different functional groups interact in a homogeneous environment. By combining theoretical analysis with experiments we could also disentangle the relationship between species richness and functional diversity, showing that functional diversity per se is a crucial driver of productivity and stability in multispecies community.
\end{abstract}

Key words: biodiversity-ecosystem functioning; community assembly; community dynamics; ecological networks; functional diversity; interaction experiment; interaction matrix; interaction strength; protist microcosm; protists; stability.

\section{INTRODUCTION}

A fundamental goal of community ecology is to investigate and understand the mechanisms that promote species coexistence and that maintain stability of biological communities (May 1972, Chesson 2000, Levine and HilleRisLambers 2009, Allesina and Tang 2012, Cardinale et al. 2013, Donohue et al. 2013, Loreau and de Mazancourt 2013, Turnbull et al. 2013). The understanding of the stability of species coexistence is central, because it is directly related to the persistence of a system over time (McCann 2000, Ives and Carpenter 2007), and to important ecosystem functions, such as productivity (Loreau et al. 2001).

Theory predicts that species in randomly assembled communities experience strong competition (Bastolla et

Manuscript received 11 July 2014; revised 10 October 2014; accepted 15 October 2014. Corresponding Editor: H. Hillebrand.

${ }^{6}$ E-mail: fcarrara@mit.edu al. 2005), increasing the likelihood of community instability (May 1972). Theoretical work has shown that both properties of the interaction matrix and species' ecological traits influence community stability and the maximum number of species within a trophic level (MacArthur and Levins 1967, Kokkoris et al. 2002, Bastolla et al. 2005). Therefore, both the architecture of ecological networks and the distribution of interaction strengths impact ecosystem stability, suggesting the presence of nonrandom assembling rules in natural ecosystems (May 1972, Solè and Montoya 2001, Stouffer and Bascompte 2011, Allesina and Tang 2012, Suweis et al. 2013). Ecological stability is a multidimensional concept that has been studied extensively for decades (see Donohue et al. [2013] for a review of this body of literature). Previous works have focused on different components of stability such as asymptotic stability (May 1972), coefficient of variation in biomass production (Tilman et al. 1998), stability at the single population level, and the likelihood of species persistence in the community. Overall, many mechanisms 

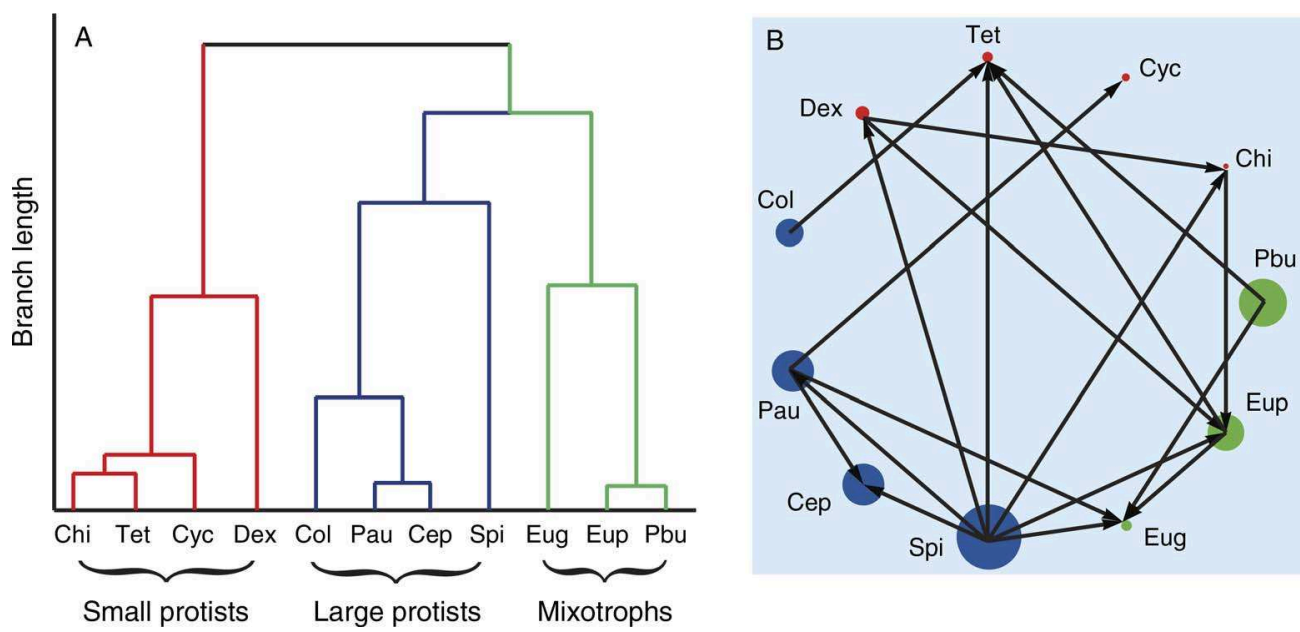

FIG. 1. Community dendrogram and competitive exclusion dynamics for the 11 protist species. (A) Community dendrogram is based on intrinsic growth rate, body size, and ability to photosynthesize as species traits. (B) The adjacency graph shows competitive exclusion dynamics in the interaction experiments. Arrows point from the excluded species to the superior competitor (six extinction events over the six replicates). Circle size is proportional to the species' body size (abbreviations of the 11 species are explained in Material and methods). Different colors are associated with different functional groups (red, small bacterivorous protists, Chi, Cyc, Tet, and Dex; blue, large bacterivorous protists, Col, Pau, Cep, and Spi; green, mixotrophic protists, Eug, Eup, and $\mathrm{Pbu}$ ). Interestingly, no loops due to intransitivity in competitive interactions (e.g., rock, paper, scissors) were detected, reflecting a high transitivity of the interaction matrix.

have been suggested as explanations for the diversitystability and diversity-functioning relationships (McCann 2000, Ives et al. 2005, Ives and Carpenter 2007, Hillebrand and Matthiessen 2009). These include portfolio (Tilman et al. 1998) and sampling effects (Loreau 1998), niche partitioning (Finke and Snyder 2008, Cardinale 2011), apparent mutualism induced by predation (Saleem et al. 2012), functional (Tilman et al. 2001), and phylogenetic diversity (Cadotte 2013). To test theoretical predictions on the relationship between diversity, stability, and productivity of biological communities, a large number of experiments have been performed with interacting species in controlled environments, including a variety of study organisms, such as plants (Tilman et al. 2001, Allan et al. 2011, Cadotte 2013), insects (Ruesink and Srivastava 2001), and algal (Bruno et al. 2006, Cardinale 2011) and microbial communities (McGrady-Steed et al. 1997, Fox and McGrady-Steed 2002, Foster and Bell 2012). However, some experimental results provide contradictory evidence (Ives and Carpenter 2007, Hillebrand and Matthiessen 2009), and most of these studies focused on a single trophic level (but see McGrady-Steed et al. 1997, Fox and McGrady-Steed 2002). By focusing on one trophic level, competition for the same resources gained the most attention (Loreau et al. 2001, Ives et al. 2005). In natural communities, however, many other kinds of species interactions exist, like predator-prey and host-parasite interactions, interference (Amarasekare 2002) or apparent competition, or positive relationships, like mutualism or cooperation (Freilich et al. 2011, Suweis et al. 2013). An assessment of the mechanisms promoting stability and productivity in trophically structured communities of functionally diverse species is thus needed (Duffy et al. 2007, Haddad et al. 2011, Faust and Raes 2012).

We conducted interaction experiments by using aquatic microcosms composed of 10 protist and one rotifer species that belong to three functional groups (small bacterivorous, large bacterivorous, and mixotrophs). We used three crucial ecological traits of our study species (intrinsic rate of growth, body size, and ability to photosynthesize) to build a functional dendrogram (Fig. 1A, see Material and methods). Our reference of functional groups is broader than commonly applied classifications, such as grouping of plants into trees, shrubs, and herbs, or into grasses, herbs, and legumes (Tilman et al. 2001, Allan et al. 2011). While such groupings of plants are clearly different, they nevertheless cover one trophic level, and are generally competing for the same macronutrients and water (Loreau et al. 2001). In our experiments, which included species varying in size across several orders of magnitude (Giometto et al. 2013), large bacterivorous may also consume smaller protists. As such, our experimental microcosm communities covered multiple trophic levels (different bacteria species feeding on common resources, all protists feeding on bacteria, with larger protists feeding also on smaller protists). We conducted interaction experiments for species monocultures, for all possible 55 pairwise combinations, and for all the 11 species interacting. From the pairwise experiments, we characterized the interaction matrix in a generalized Lotka-Volterra (LV) framework, to single out key processes driving community complexity (Vandermeer 1969). We generated numerical simulations using a 
stochastic LV community model, parametrized with the pairwise experimental interaction strengths of the interaction matrix. At the same species richness level, communities with different functional diversity were randomly initialized.

Here, we addressed the question of how species' traitrelatedness, functional diversity and properties of the interaction matrix affect coexistence and community productivity. Moreover, through our approach that combined experimental with theoretical results, we could disentangle the relationship between species richness and functional diversity. Taken together, our results suggest that functional diversity is a crucial driver of productivity and stability in multispecies community.

\section{Material and Methods}

\section{Aquatic communities}

We used a pool of 10 protist and one rotifer species in the experiments (henceforth called protists). The species were: Chilomonas sp. (Chi), Colpidium sp. (Col), Cyclidium sp. (Cyc), Dexiostoma sp. (Dex), Euglena gracilis (Eug), Euplotes aediculatus (Eup), Paramecium aurelia (Pau), P. bursaria (Pbu), Spirostomum sp. (Spi), Tetrahymena sp. (Tet), and the rotifer Cephalodella sp. (Cep). Four species, Chi, Cyc, Dex and Tet, were bought at Carolina Biological Supply (Burlington, North Carolina, USA), whereas all other species were originally isolated from a natural pond (McGrady-Steed et al. 1997). All these species can naturally co-occur in freshwater habitats and form natural trophically structured communities/food webs (McGrady-Steed et al. 1997, Mächler and Altermatt 2012). Furthermore, such microbial organisms drive the bulk of ecosystem processes and cover substantial biological complexity in terms of ecological traits, such as nutrient uptake, photosynthetic capabilities or swimming ability, species interactions, and trophic levels, and thereby offer ideal study systems (Jessup et al. 2004, Altermatt et al. 2011). We grew the species in sterilized culture medium made of local spring water and $0.45 \mathrm{~g} / \mathrm{L}$ of Protozoan Pellets (Carolina Biological Supply). Protozoan Pellets provide nutrients for three species of bacteria (Breviacillus brevis, Bacillus subtilis, and Serratia fonticola) added to the cultures. All of the herein used species can feed on bacteria. However, they may prefer different bacteria species, depending on morphology, size (DeLong and Vasseur 2012), or phylogeny of both protists and bacteria (Glücksman et al. 2010). Local communities of these species were maintained in culture well plates containing $10 \mathrm{~mL}$ of culture medium. We conducted all experiments in a climatized room at $20^{\circ} \mathrm{C}$ under constant fluorescent light (for method details, see also Altermatt et al. 2011, Carrara et al. 2012, 2014).

\section{Species' traits: functional diversity}

We used intrinsic rate of growth, body size, and ability to photosynthesize to build a functional dendrogram (Petchey and Gaston 2002), in which species are assigned to the tips of the dendrogram (Fig. 1A). The intrinsic growth rate of our species reflects fitness differences, while body size and ability to photosynthesize as further species traits impact the overlap of resource use between species. We used values of intrinsic growth rates, body size, and ability to photosynthesize of all study species from Carrara et al. (2012, 2014), and Giometto et al. (2013). In these studies, the same protist species were cultivated at identical conditions as used for the interaction experiment in the current study. We calculated functional diversity for a given community by summing the distance along the branches, from the occupied tips to the top of the dendrogram (Petchey and Gaston 2002). We adopted a broad definition of functional diversity, such that it also included different trophic groups. At the highest hierarchical level of the community dendrogram, we defined functional groups by grouping species that belonged to the same branch (Fig. 1A). Thereby Chi, Cyc, Tet, and Dex were forming a functional group of small bacterivorous species (body size ranging from 0.6 to $4.5 \times 10^{-6} \mathrm{~g}$ ) with high growth rates $\left(r>1.5 \mathrm{~d}^{-1}\right)$. Col, Pau, Cep, and Spi formed a group of large bacterivorous species (body size ranging from 20 to $1000 \times 10^{-6} \mathrm{~g}$ ). Furthermore, these four species may not only feed on bacteria, but may also predate directly on smaller protist species, such as Chi, Cyc, Tet, and Dex, as well as on microflagellates, which are always present in such cultures (Altermatt et al. 2011) and remained unidentified. Among the larger bacterivorous species, Col is feeding essentially on bacteria species, whereas Cep (the rotifer) and Spi are known to consume also smaller protists. Finally, Eug, Eup, and $\mathrm{Pbu}$ formed a group of mixotrophic species that are all capable of photosynthesizing. Among the mixotrophs, Eug is mostly an autotroph species, whereas Eup may feed substantially on bacteria species. We acknowledge that the diet specifications of these multi-trophic groups are somewhat flexible and thus the groups could also be referred to as multi-functional groups.

\section{The interaction experiments}

We performed interaction experiments in microcosm communities of our 11 protist species, belonging to the above-described three functional groups. We measured population growth of all species in isolation, all possible 55 pairwise species combinations as well as the 11species combination. To set up the experiment, we first grew all 11 species in isolated cultures to carrying capacity and measured their densities $\left(\phi_{i}^{0}, \phi_{j}^{0}\right)$. Then, we mixed $5 \mathrm{~mL}$ of medium of species $i$ at carrying capacity to $5 \mathrm{~mL}$ of species $j$ at carrying capacity (the total volume in each community, $V$, was $10 \mathrm{~mL}$ ). Speciesspecific carrying capacities, which scale with body size (see Appendix A: Fig. A1; White et al. 2007), were measured in pure cultures in a control experiment. In parallel, we also measured the species' ability to coexist in communities that were composed of all 11 protist 
species. For this part, we initialized the microcosms with $V / 11$ volume of medium of each species culture grown to carrying capacity, while the experimental treatment was otherwise identical to the pairwise species combinations and also conducted simultaneously. Position of all replicates (pairwise and 11-species communities) was randomized in our climate chamber.

We replicated the 11 one-species, the 55 two-species, and the one 11-species communities six times each (i.e., in total 402 replicates). We measured the density of each species in all microcosms after three weeks (i.e., at $t^{*}=$ $21 \mathrm{~d}$ ). We sampled and counted densities of species in a variable quantity of medium optimized for each specific species (Altermatt et al. 2011) under a stereo microscope. In addition to the above-described interaction experiment in which we took one abundance measurement after three weeks, we measured time-series data on selected two-, three-, and four-species communities. Specifically, we obtained time series (all six times replicated and sampled for at least 10 days) for the following two-species combinations: Col-Cep, Col-Tet, Cep-Tet. We also obtained time series for the threespecies community Col-Cep-Tet and for the fourspecies community Col-Eug-Cep-Tet (Appendix B: Fig. B1). We thereby implemented a gradient in functional diversity level and species richness on a subset of all 11 species at identical experimental conditions.

\section{Community model}

In LV models, the dynamics of species $i$ and species $j$ are characterized by phenomenological equations with linear interaction terms. Generalizing the two-species LV model to a community with $S=11$ species, a system of coupled differential equations is derived (see, e.g., Kokkoris et al. 2002), where density changes of species $i$ are described by

$$
\frac{d \phi_{i}}{d t}=r_{i} \phi_{i}\left(1+\frac{\alpha_{i i} \phi_{i}+\sum_{j \neq i} \alpha_{i j} \phi_{j}}{K_{i}}\right)
$$

where $\phi_{i}(t)=\left\langle N_{i}(t)\right\rangle / V$ is the population density of species $i, r_{i}$ its intrinsic growth rate, $K_{i}$ its carrying capacity, $\alpha_{i j}$ measures the strength of interspecific competition between $i$ and $j$, and $\alpha_{i i}$ measures the intraspecific competition. The values $\alpha_{i j}$ for all pairwise $i$ and $j$ constitute the interaction matrix, A. After rescaling the density of species $i$ by its carrying capacity (Kokkoris et al. 2002), $K_{i}, n_{i}=\phi_{i} / K_{i}, \alpha_{i j}^{\prime}=\alpha_{i j} K_{j} / K_{i}$ the LV model becomes

$$
\frac{d \mathbf{n}}{d t}=\mathbf{r n}\left(1+\mathbf{A}^{\prime} \mathbf{n}\right)
$$

where $\mathbf{A}^{\prime}$ is the experimental interaction matrix (rescaled to each species' carrying capacity), $\mathbf{r}=\left(r_{1}, r_{2}, \ldots, r_{11}\right)$, and $\mathbf{n}=\left(n_{1}, n_{2}, \ldots, n_{11}\right)$. We investigated the relationship between functional diversity and species coexistence, community stability, and productivity through simulations by using the interaction matrix $\mathbf{A}$ obtained in the pairwise experiments. The number of possible species combinations from our species pool, $S=11$, at each richness level, $k$, increases as the binomial coefficient

$$
\left(\begin{array}{c}
11 \\
k
\end{array}\right)
$$

and performing experiments for every combination becomes prohibitive. Thus, through our model, we could consistently explore a much wider solutions space, and thereby complementing and generalizing the experimental results. As such, the goal of the model is not necessarily to match the experimental results within the tested species combinations, but to get a qualitative understanding of dynamics in communities with 5-10 species present. Simulations covered the entire range of species richness (from to 2 to 11 ), preserving the proportion of different combinations (the binomial coefficient) at each richness level. We ran simulations for initial communities composed of species belonging to one functional group only (for each functional group separately), two functional groups (three functional groups combinations), and three functional groups (the 11-species experiment). In the 11-species simulations, all species were present with a known initial density, as in the main experiment. As such, communities with different functional diversity were randomly initialized at each richness level. We quantified productivity as total biomass production at the end of simulations. Stability was captured by the coefficient of variation of community biomass (CV). Both these metrics have been widely adopted in community ecology to study diversityproductivity and diversity stability relationships (Cardinale et al. 2013). We recognized that community stability (herein measured as the coefficient of variation in biomass production) represents only a single component of stability, a multidimensional concept (see Donohue et al. [2013] for an extensive review of this body of literature).

All simulations were run over 21 days, that is, the experimental duration, and $\mathrm{CV}$ was measured over days 11-21 of each simulation. We employed a Gillespie algorithm (Gillespie 1977) to directly solve the master equation associated to the deterministic system of equations (2), thereby including demographic stochasticity (for a stochastic formulation of this model, see Carrara et al. 2012).

\section{Species interaction type}

Species interactions $\alpha_{i j}$ were derived by fitting the time series of the two-species interaction to a LV model, constrained to the initial conditions adopted in the experiment, $\phi_{i}^{0}, \phi_{j}^{0}$, and the final densities $\phi_{i}^{*}, \phi_{j}^{*}$ at $t^{*}$. The generalized LV modelling approach in principle includes predator-prey dynamics and positive interactions. Species with competitive interactions have negative $\alpha$ values. The sign of $\alpha$ is positive when a predator- 
prey or mutualistic interaction is occurring between two species. A predator-prey interaction $i-j$ has $\alpha_{i j} \alpha_{j i}<0$. In mutualistic interactions, both $\alpha_{i j}$ and $\alpha_{j i}$ are positive $\left(\phi_{i}^{*}\right.$ $>K_{i}, \phi_{j}^{*}>K_{j}$ ). Amenalism/commensalism arises when one value of $\alpha$ is equal to zero and the other is negative/ positive, respectively. Non-interacting species have both $\alpha$ values equal to zero. The interaction type was assigned for each species pair using the above-described categories, considering the experimental errors (SE calculated from the six replicates) associated with each $\alpha$ value. Differences in intra- vs. inter-group distributions of species interaction strength were tested with a Kolmogorov-Smirnov test on the cumulative distributions. We built adjacency graphs that are reflecting (competitive) exclusion dynamics between the 11 protist species measured in the interaction experiments. The arrows in the graphs point from the excluded species to the superior competitor (as it is the convention in food-web literature for predator-prey links). A deterministic extinction of an inferior species caused by the superior species occurred for six extinction events over the six replicates. We detected the presence of competitive or mutualistic loops within interactions among protist species, where such loops reflect the degree of intransitivity in competitive interactions (Allesina and Levine 2011). A set of species defined a mutualistic loop when each species was favoring another species forming a closed chain of positive species interactions.

\section{Community productivity and additivity}

Productivity (i.e., biomass yield) was obtained by multiplying the number of individuals in a community times the average cell size. Biomass is always expressed in $\mathrm{g} / \mathrm{mL}$, obtained from direct measurements of body volume/size using a particle counter, assuming a constant density equal to the density of water and thereby not making a distinction between body volume and body mass (data after Giometto et al. [2013], at identical environmental conditions). For the one-species communities, $Y_{1}$ is the average biomass yield obtained in monocultures; for the two-species setup, $Y_{2}$ is the average biomass yield obtained in all the 330 microcosms of the pairwise interaction experiment; for the 11species setup, $Y_{11}$ is the average over the yields of the six replicates. An additive partitioning analysis was conducted to calculate the total biodiversity effect in terms of complementarity and selection effects. The selection effect (SE) is measured by the covariance between the monoculture yield of species and their change in relative yield in the multispecies. The complementarity effect (CE) is measured by the difference between the average species yields in the mixture and the weighted average monoculture yield of the component species (Loreau and hector 2001). Positive complementarity effects occurs when species on average have higher than expected relative yield (RY), where the expected RY is that each species will produce biomass in proportion of the number of species in the community. A positive selection effect indicates that there is a positive covariance between relative yield and one-species biomass.

In order to decouple the effect of abundance and species body size on community productivity, results were also compared in terms of biomass relative to each species' monoculture, by rescaling to its carrying capacity, $n_{i}^{*}=\phi_{i}^{*} / K_{i}$. Thus, we could consider the sum of species relative yields in the community rescaled to the number $S$ of species in the community, $A_{S}=\left(\sum_{i=2}^{S} n_{i}^{*}\right) / S$, as a measure of additivity in an $S$-species community. A complete additive community would give a value of one. Additivity was calculated for the two-species communities $\left(A_{2}\right)$, differentiating for intra- $\left(A^{\text {intra }}\right)$ and inter-group species $\left(A^{\text {inter }}\right)$, and for the 11-species community setup $\left(A_{11}\right)$.

\section{RESUlts \\ Experimental results}

Species interaction type.-We observed a total of 207 population extinctions in the $330(55 \times 6)$ microcosms, with initially 660 starting populations. The majority of observed pairwise interactions were of competitive nature (56\%). Furthermore, predator-prey and amenalistic interactions constituted $26 \%$ and $18 \%$ of the total number of interaction types. No neutral, commensalistic, or mutualistic interactions were found (Fig. 2). No competitive loops were found among the 11 protists species (Fig. 1B), meaning that communities manifested a high degree of transitivity (Appendix C: Fig. C1).

When analyzing species interactions in relation to functional groups, we detected a compartmentalization of the interaction matrix (Fig. 2B) that resulted in significantly different interaction-strength distributions of intra- vs. inter-group interactions (KolmogorovSmirnov test, $P=0.005)$. More similar species, belonging to the same functional group, competed more strongly than species from different groups. Intra-group interactions were dominated by competitive interactions $(80 \%)$, with very few predator-prey links $(6 \%)$, showing higher competitive strengths (Fig. 2C, D). Inter-group interactions showed a weaker majority of competitive interactions (less than 50\%), with strong predator-prey dynamics (33\%), resulting in a more skewed distribution (Fig. 2C, D). We detected mutualistic loops, but only between species in different functional groups (Appendix C: Fig. C2).

Community productivity and additivity.-Community biomass productions in one-species populations were not statistically different from two-species communities $\left(Y_{1}=5.5 \times 10^{-3} \mathrm{~g} / \mathrm{mL}, Y_{2}=5.5 \times 10^{-3} \mathrm{~g} / \mathrm{mL}, t\right.$ test, $t_{10}=$ $0.01, P=0.99$, Fig. 3). By applying additive partitioning to the two-species communities, we found a balance between a positive selection effect $\left(\mathrm{SE}_{2}=2 \times 10^{-4} \mathrm{~g} / \mathrm{mL}\right)$ and a negative complementarity effect $\left(\mathrm{CE}_{2}=-1.7 \times\right.$ $10^{-4} \mathrm{~g} / \mathrm{mL}$ ). In the 11 -species communities, average community biomass, $Y_{11}$, was significantly higher than both $Y_{1}$ and $Y_{2}\left(Y_{11}=0.0429 \mathrm{~g} / \mathrm{mL}\right.$, Fig. $3, t_{10}=1.3, P$ 

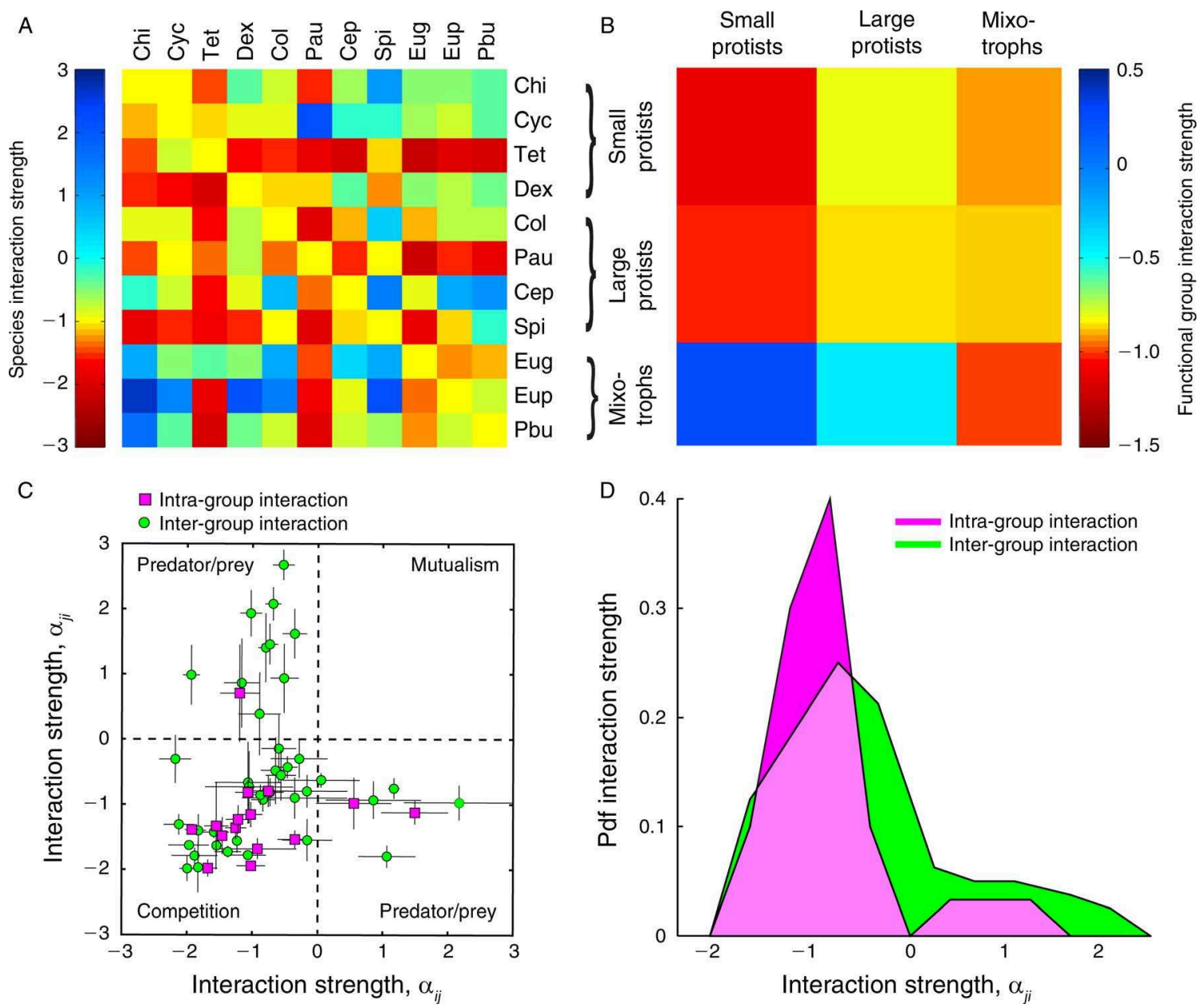

FIG. 2. Experimental results on the interactions between the 11 protist species. (A) Interaction matrix describing all experimentally measured pairwise interaction strengths between the 11 species. Species are ordered according to three functional groups (small bacterivorous protists, large bacterivorous protists, mixotrophic protists). The color of the square at position $i, j$ indicates the effect of species $j$ on species $i$. (B) Average interaction strength within each functional group between species belonging to different functional groups. (C) Interaction strength $(\alpha)$ distribution, separately given for species belonging to the same functional groups (intra-group, magenta), and to different groups (inter-group, green). Error bars show \pm SE and represent the uncertainty associated with each $\alpha$ value. (D) Probability density function (pdf) of intra-group (magenta) and inter-group (green) interaction strengths (lighter pink color where distributions overlap). In a Lotka-Volterra model, the interaction coefficients are captured by constant $\alpha$ values, describing the effect of species $j$ on species $i\left(\alpha_{i j}\right)$ and the effect of species $i$ on species $j\left(\alpha_{j i}\right)$.

$<10^{-5}$ ), due to a strong positive complementarity effect $\left(\mathrm{CE}_{11}=3.98 \times 10^{-2} \mathrm{~g} / \mathrm{mL}\right)$ and a moderate selection effect $\left(\mathrm{SE}_{11}=3.1 \times 10^{-3} \mathrm{~g} / \mathrm{mL}\right)$. These patterns were confirmed at lower richness values, where we followed a subset of species over time (two to four species from the whole pool, Appendix B: Fig. B1). Complementarity and selection effects were comparable in the two-species communities, with low level of functional diversity $\left(\mathrm{CE}_{2}\right.$ $=0.0028 \mathrm{~g} / \mathrm{mL}, \mathrm{SE}_{2}=0.0013 \mathrm{~g} / \mathrm{mL}$; Fig. B1a-c). In the three-species combination, $\mathrm{CE}$ was four times greater than $\mathrm{SE}\left(\mathrm{CE}_{3}=0.0107 \mathrm{~g} / \mathrm{mL}, \mathrm{SE}_{3}=0.0025 \mathrm{~g} / \mathrm{mL}\right.$; Fig. B1) and in the four-species combination, where four species from three different functional groups were grown together, $\mathrm{SE}$ was negative $\left(\mathrm{CE}_{4}=0.0106 \mathrm{~g} / \mathrm{mL}\right.$, $\mathrm{SE}_{4}=-0.0007 \mathrm{~g} / \mathrm{mL}$; Fig. B1e).
In the two-species communities, an additivity measure for relative yields gives $A_{2}=0.41$, and intra-group additivity was higher than inter-group additivity $\left(A_{2}^{\text {intra }}=\right.$ $0.27, A_{2}^{\text {inter }}=0.47$ ).

Additivity in 11 -species communities was higher $\left(A_{11}\right.$ $=0.69$ ) compared to two-species communities (inset Fig. 3 , a pure additive community would give $A=1$, see also Appendix D: Fig. D1).

\section{Theoretical simulations}

Community properties in a functional diversity gradient.-A positive relationship between functional diversity and total biomass production was found in our community model parametrized by the pairwise experimental interaction strengths of the interaction matrix (Fig. 4A), confirming the experimental results. Commu- 


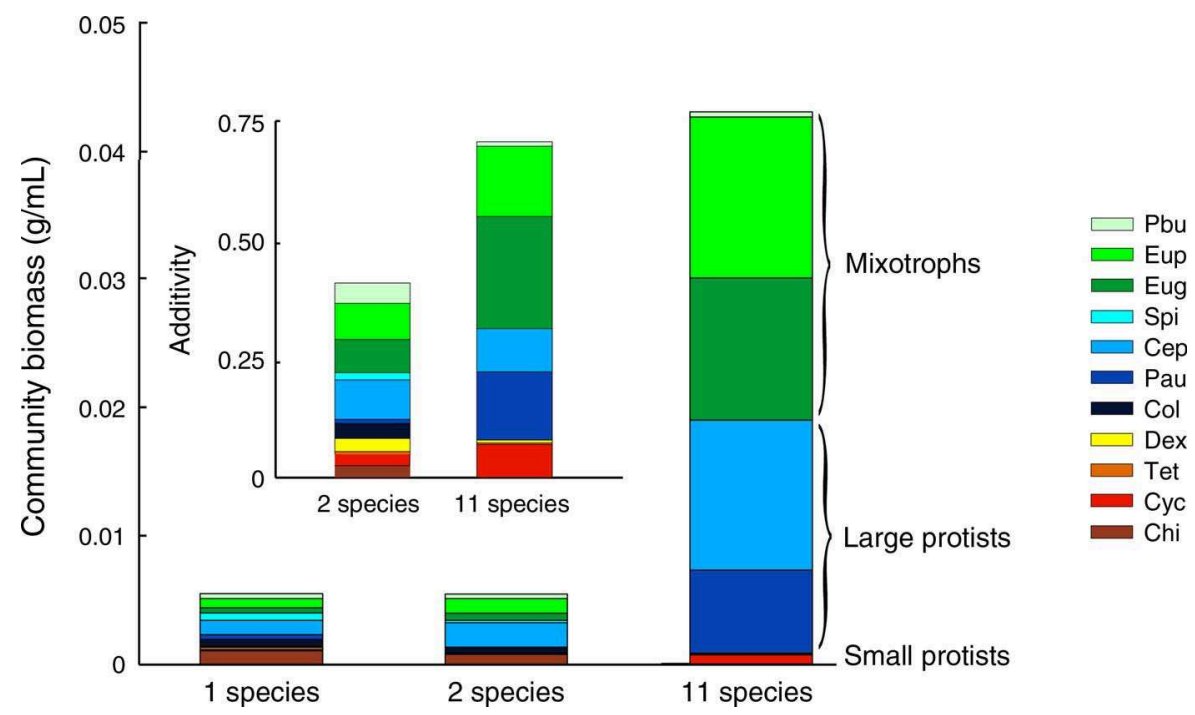

FIG. 3. Biomass production in 1-species, 2-species, and 11-species communities. All values represent the average over six experimental replicates (the 2 -species communities are averaged over the 55 combinations). Inset: additivity shows community productivity rescaled to each species' carrying capacity. A complete additive community would result in a value of one (all experimentally observed values are lower). Species order is the same as in Fig. 2A. Species belonging to the same functional group share similar colors (red and yellow colors, small bacterivorous protists; blue colors, large bacterivorous protists; green colors, mixotrophic protists).

nities composed of species from one or two functional groups were less productive compared to communities where species belonged to three functional groups (Fig. 4B, Appendix E: Fig. E1). Moreover, we observed a higher final species richness for communities with a higher initial functional diversity when looking at species coexistence of communities initially composed of the same level of species richness, but across a functional diversity gradient (Fig. 5A). This pattern is reflected in higher community productivity (total biomass production; Fig. 5B) and a generally higher stability (coefficient of variation of community biomass over time; Fig. 5C), when the initial community was composed of more functionally diverse species.

\section{Discussion}

By combining experimental results (Figs. 2 and 3, and Appendix B: Fig. B1) and theoretical models (Figs. 4 and 5, and Appendix E: Fig. E1), we showed the importance of high levels of functional diversity on the stability of species coexistence and on total biomass production in multispecies communities. In line with our findings, recent empirical observations on biodiversityecosystem functioning (BEF) suggest a positive effect of diversity on community stability and productivity (McGrady-Steed et al. 1997, Ruesking and Srivastava 2001, Tilman et al. 2001, Allan et al. 2011, Foster and Bell 2012, Cadotte 2013). Toward a mechanistic understanding of our findings in this context, we suggested a way to disentangle the relative contribution of species richness and functional diversity on community composition (Figs. 4 and 5). We found that the presence of species from different functional groups increased the productivity of species in the community compared to communities of similar initial diversity that lacked a functional differentiation, supporting previous findings (Tilman et al. 2001, Heemsbergen et al. 2004, Griffin et al. 2008). Our results are deemed especially important, because the functional groups used in our study system covered multiple trophic levels and a wide range in size and phylogenetic history (including Alveolata, Chloroplastida, and Metazoa [Adl et al. 2012, Giometto et al. 2013]).

Experimental and theoretical evidence on how changes in diversity on a particular trophic level affect the whole food-web (Solè and Montoya 2001, Haddad et al. 2011), calls for analyses incorporating several trophic levels simultaneously (Duffy et al. 2007). When studying the effects of diversity on community stability or productivity, experiments were primarily conducted with plants or grassland systems, belonging to different functional groups (Tilman et al. 2001, Levine and HilleRisLambers 2009, Allan et al. 2011, Cadotte 2013). Functional groups within plants are still restricted to the use and competition for the same resources (such as macronutrients and water). Natural communities, however, are mostly (if not always) composed of functionally more dissimilar groups (including heterotrophs, autotrophs, and predators). Therefore, conclusions from competition within different functional groups in plants cannot be directly compared to wider ranges of functional groups. In our experiments, we extended from previous studies by observing dynamics in a complex food web, with multiple trophic levels. Furthermore, our measure of functional diversity was directly associated to ecological traits that were exper- 

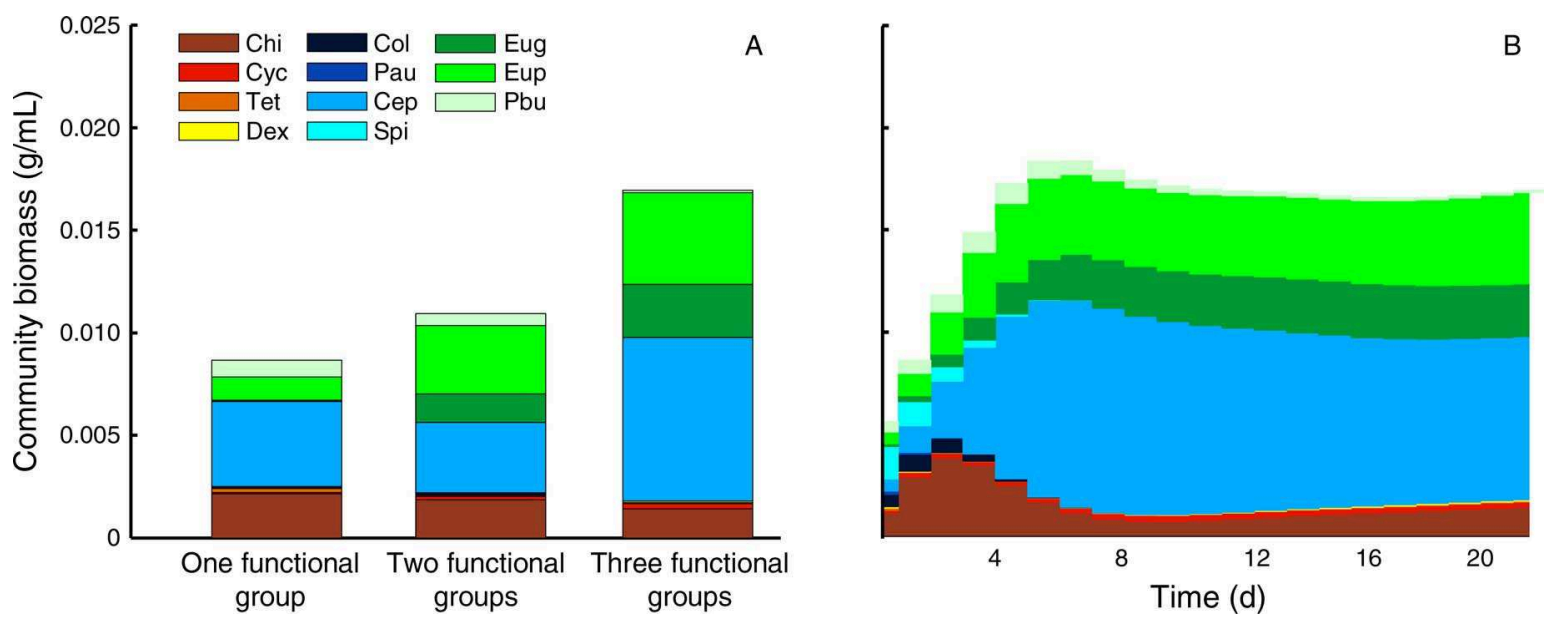

FIG. 4. Relationship between total biomass production and functional groups based on simulations adopting a generalized Lotka-Volterra model. (A) Total biomass production in a gradient of functional diversity (increasing number of functional groups). The left and central bars correspond to communities with species from one and two functional groups, respectively (averages of panels (A-C) and (D-F) of Appendix E: Fig. E1). The values are the average community biomass productions over simulations for different combinations of species. (B) Time series plot for the 11-species communities composed by the three functional groups. The interaction matrix in the model is parametrized with the empirical data from the pairwise-interaction experiment (Fig. 2A).

imentally observed. Thereby, our analysis could investigate diversity-stability and diversity-productivity relationships from a more quantitative perspective compared to previous microbial studies, in which a detailed quantification of functional diversity in terms of ecological traits was lacking (McGrady-Steed et al. 1997, Fox and McGrady-Steed 2002).

Our pairwise interaction experiments showed higher competitive strengths among species sharing similar ecological traits (Fig. 2), as recently found in microbial communities using protists (Violle et al. 2011). However, the architecture in the interaction matrix showed a compartmentalization between species belonging to different functional groups (Stouffer and Bascompte 2011). This resulted in a left skewed distribution for the inter-group interactions (Fig. 2D) that stabilized the communities (Allesina and Tang 2012). The presence of species belonging to different functional groups (e.g., mixotrophs and predators) led to increased coexistence of the more related species within the individual groups in multispecies communities (i.e., increased coexistence within mixotrophs and predators; Appendix B: Fig. B1). This higher species coexistence enhanced productivity in multispecies communities compared to the average onespecies and two-species communities (Fig. 3, Appendix D: Fig. D1). Thus, our results suggest that the high dissimilarity across functional groups promotes coexistence and productivity that would be prevented in functionally less diverse communities (but characterized by identical species richness).

We detected the presence of strong complementarity forces (Loreau and Hector 2001) due to niche differences
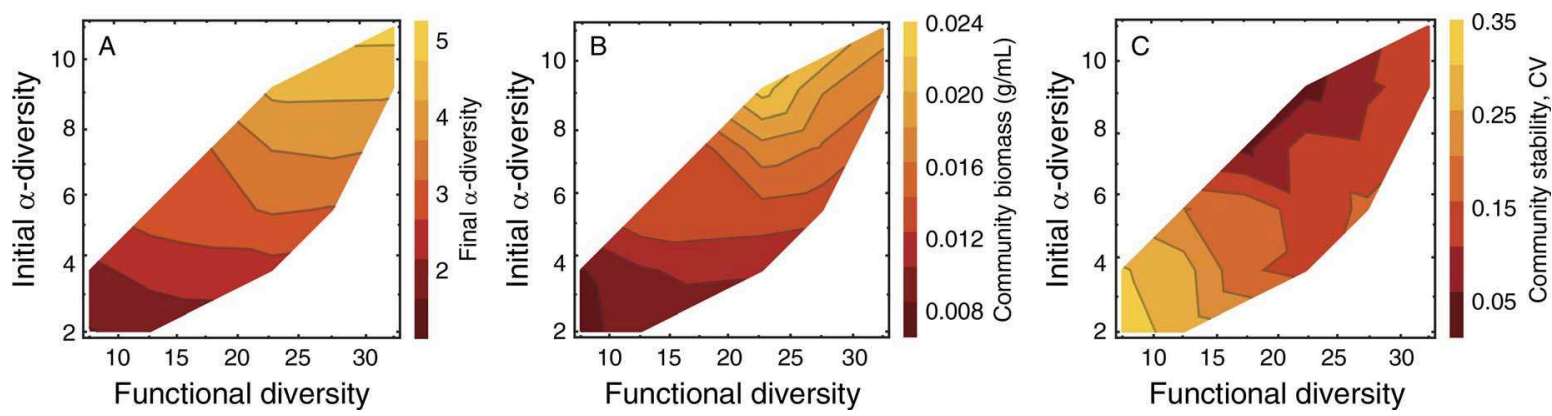

FIG. 5. Impact of functional diversity on community properties. Color gradients show (A) realized final $\alpha$-diversity, (B) total biomass production, and $(\mathrm{C})$ coefficient of variation at the end of simulations adopting a generalized Lotka-Volterra model. The colored domain represents the feasible region with the lowest/highest functional diversity ( $x$-axis), at each level of species richness ( $y$-axis). The simulations are based on initially identical communities with respect to species richness (2-11 species), but at different levels of functional diversity. The interaction matrix in the model is fitted on the pairwise interaction experiments (Fig. $2 \mathrm{~A}$, as for Fig. 4). Communities with higher functional diversity have generally greater productivity and statistical stability (negative slopes of the isoclines). At the highest values of initial species richness, there is redundancy in functional diversity and the curves start to saturate. 
in resources use (Finke and Snyder 2008) in communities composed by functionally dissimilar species. Our results generalize previous findings of complementarity effects in trophically structured communities (Finke and Snyder 2008, Levine and HilleRisLambers 2009, Cardinale 2011). We also found a higher additivity for functionally more diverse communities at different degrees of species richness (inset of Fig. 3). However, the increase in additivity, when all species were growing together, was less than the total biomass of all species when grown in isolation (additivity $A<1$ ). This echoes recent experimental findings with single trophic level bacterial communities (Foster and Bell 2012). Specifically, it suggests a non-additive effect of the total biomass production along a species richness gradient. A complete additive model, assuming no overlaps of species' niche, would represent an unrealistic assumption for our model system, as all protists species feed on the same bacteria. Furthermore, precise allometric relationships dictate the species' diet with respect to what bacteria they can feed on (DeLong and Vasseur 2012), which implies overlaps in resource use for our protist species.

In our experimental model system, large bacterivorous species were regularly and directly predating on smaller protists belonging to the first functional group. The interaction between competition for bacteria (the common food resource) and intraguild predation (Finke and Denno 2005), which takes place at similar timescales (Chesson and Kuang 2008), may have played a role in determining the experimental outcome in the 11-species community, as recently found by experiments on bacteria species (Saleem et al. 2012). Moreover, the natural variability in intrinsic growth rates, resulting in high functional diversity, allows species from different functional groups to have differential responses over time (Tilman et al. 1998), eventually favoring coexistence (Allan et al. 2011), and enhancing ecosystem predictability (McGrady-Steed et al. 1997). By these mechanisms, species result in more effective intraspecific and intra-group interactions compared to interspecific and inter-group interactions (Fig. 2; Levine and HilleRisLambers 2009) thus recovering in a community when at low numbers. On the contrary, communities composed of species within the same functional group, where stabilizing forces are generally weaker, and intrinsic growth rates are similar (Chesson 2000), tend to be more unpredictable (Ives and Carpenter 2007). Stochastic effects may play a stronger role in community assembly for species with similar ecological traits or that are closely related. Stronger priority effects for phylogenetically related species were observed in experiments on microbial communities (Peay et al. 2012, Tan et al. 2012). In our simulated communities, a small differentiation in functional traits resulted in a decrease of the system's stability (Fig. 5C), due to the emergence of multiple domains of attraction, where dynamics are strongly dependent on the initial conditions. Interestingly, the effects of functional diversity on the stability of species coexistence and productivity are more pronounced at lower levels of diversity, compared to communities initially composed by higher levels of initial species richness. This may be related to a redundancy in functional differentiation of the species interacting in the community (Fig. 5).

From the pairwise experimental results, we found a high degree of transitivity among the 11 species as no competitive loops were detected (Fig. 1b, Appendix C: Fig. C1). In fact, competitive loops reflect the degree of intransitivity in competitive interactions (Allesina and Levine 2011). The transitivity, therefore, may explain the general agreement between an additive model fitted on the pairwise interactions and the experimental results of the 11-species community. Even though the additive LV model showed results consistent with the experimental findings (Vandermeer 1969), it cannot capture non-additive effects, relative non-linearities in intrinsic growth rate (Chesson 2000), or other forms of positive or negative interactions, such as interference competition (Amarasekare 2002) or prey switching (Glücksman et al. 2010), which likely occurred in our experimental communities. It is also likely that competition dynamics at lower trophic levels (bacteria and microflagellates) may have consequences for protists' dynamics (Chesson and Kuang 2008). P. aurelia, which appeared as a bad competitor in the pairwise rounds, routinely flourished in the 11-species community (Fig. 3). More complex dynamics, such as non-additive effects, may emerge when multiple agents interact (Vandermeer 1969, Case and Bender 1981). Interestingly, through our approach, we detected mutualistic loops between triplets of species belonging to three different functional groups (Fig. C2), proving the existence of positive non-additive effects for certain species in functionally diverse communities, which were instead not detected in pairwise competition rounds.

In conclusion, we showed that in communities composed of multiple trophic groups with functionally dissimilar species, the variety of species interactions promotes both stability and biomass production at the community level. Thus, looking at species richness only as a proxy of diversity of a community may be misleading. The inclusion of trophic trait structure and functional diversity has important implications for conservation strategies and ecosystems managements as it is needed to predict important properties of communities, namely their stability and productivity.

\section{ACKNOWLEDGMENTS}

We are grateful to M. Gatto, M. Holyoak, J. Levine, and A. Maritan for stimulating discussions and useful suggestions. We thank R. Illi, Y. Bösch, and E. Britt for help in laboratory work. Funding from ERC Advanced Grant RINEC 22761 (A. Rinaldo, F. Carrara); SFN Grant 200021/124930/1 (A. Rinaldo, F. Carrara); SNF Grants 31003A/135622 and PP00P3/150698 (to F. Altermatt and M. Seymour); and Eawag Discretionary funds (to F. Altermatt and A. Giometto). F. 
Carrara also acknowledges the SNF Early Postdoc Mobility Fellowship. We also thank two anonymous reviewers for their helpful comments.

\section{Literature Cited}

Adl, S. M., et al. 2012. The revised classification of eukaryotes. Journal of Eukaryotic Microbiology 59:429-514.

Allan, E., W. Weisser, C. Rosher, M. Fisher, and H. Hillebrand. 2011. More diverse plant communities have higher functioning over time due to turnover in complementarity dominant species. Proceedings of the National Academy of Sciences USA 108:17034-17039.

Allesina, S., and J. M. Levine. 2011. A competitive network theory of species diversity. Proceedings of the National Academy of Sciences USA 108:5638-5642.

Allesina, S., and S. Tang. 2012. Stability criteria for complex ecosystems. Nature 483:205-208.

Altermatt, F., A. Bieger, F. Carrara, A. Rinaldo, and M. Holyoak. 2011. Effects of connectivity and recurrent local disturbances on community structure and population density in experimental metacommunities. PLoS ONE 6:e19525.

Amarasekare, P. 2002. Interference competition and species coexistence. Proceedings of the Royal Society B 269:25412550.

Bastolla, U., M. Lassig, S. C. Manrubia, and A. Valleriani. 2005. Biodiversity in model ecosystems, I: coexistence conditions for competing species. Journal of Theoretical Biology 235:521-530.

Bruno, J. F., S. C. Lee, J. S. Kersetz, R. C. Carpenter, Z. T. Long, and E. Duffy. 2006. Partitioning the effects of algal species identity and richness on benthic marine primary production. Oikos 115:170-178.

Cadotte, M. W. 2013. Experimental evidence that evolutionarily diverse assemblages result in higher productivity. Proceedings of the National Academy of Sciences USA 110:8996-9000.

Cardinale, B. J. 2011. Biodiversity improves water quality through nice partitioning. Nature 472:86-89.

Cardinale, B. J., K. Gross, K. Fritschie, P. Flombaum, J. W. Fox, C. Rixen, J. van Ruijven, M. Scherer-Lorenzen, and B. Wilsey. 2013. Biodiversity simultaneously enhances the production and stability of community biomass, but the effects are independent. Ecology 94:1697-1707.

Carrara, F., F. Altermatt, I. Rodriguez-Iturbe, and A. Rinaldo. 2012. Dendritic connectivity controls biodiversity patterns in experimental metacommunities. Proceedings of the National Academy of Sciences USA 109:5761-5766.

Carrara, F., A. Rinaldo, A. Giometto, and F. Altermatt. 2014. Complex interaction of dendritic connectivity and hierarchical patch size on biodiversity in river-like landscapes. American Naturalist 183:13-25.

Case, T. J., and E. A. Bender. 1981. Testing for higher order interactions. American Naturalist 118:920-929.

Chesson, P. 2000. Mechanisms of maintenance of species diversity. Annual Review of Ecology and Systematics 31: 343-366.

Chesson, P., and J. J. Kuang. 2008. The interaction between predation and competition. Nature 456:235-238.

DeLong, J. P., and D. A. Vasseur. 2012. Size-density scaling in protists and the links between consumer-resource interactions parameters. Journal of Animal Ecology 81:1193-1201.

Donohue, I., O. L. Petchey, J. M. Montoya, A. L. Jackson, L. McNally, M. Viana, K. Healy, M. Lurgi, N. E. O'Connor, and M. C. Emmerson. 2013. On the dimensionality of ecological stability. Ecology Letters 16:421-429.

Duffy, J. E., B. J. Cardinale, K. E. France, P. B. McIntyre, E. Thebault, and M. Loreau. 2007. The functional role of biodiversity in ecosystems: incorporating trophic complexity. Ecology Letters 10:522-538.
Faust, K., and J. Raes. 2012. Microbial interactions: from network to models. Nature Review Microbiology 10:538549.

Finke, D. L., and W. E. Snyder. 2008. Niche partitioning increases resource exploitation by diverse communities. Science 321:1488-1490.

Foster, K. R., and T. Bell. 2012. Competition, not cooperation, dominates interactions among culturable microbial species. Current Biology 22:1845-1850.

Fox, J. W., and J. McGrady-Steed. 2002. Stability and complexity in microcosm communities. Journal of Animal Ecology 71:749-756.

Freilich, S., R. Zarecki, O. Eilam, E. S. Segal, C. S. Henry, M. Kupiec, U. Gophna, R. Sharan, and E. Ruppin. 2011. Competitive and cooperative metabolic interactions in bacterial communities. Nature Communications 2:589.

Gillespie, D. T. 1977. Exact stochastic simulation of coupled chemical-reactions. Journal of Physical Chemistry 81:2340 2361.

Giometto, A., F. Altermatt, F. Carrara, A. Maritan, and A. Rinaldo. 2013. Scaling body-size fluctuations. Proceedings of the National Academy of Sciences USA 110:4646-4650.

Glücksman, E., T. Bell, R. I. Griffiths, and D. Bass. 2010. Closely related protist strains have different grazing impacts on natural bacterial communities. Environmental Microbiology 12:3105-3113.

Griffin, J. N., K. L. de la Haye, S. J. Hawkins, R. C. Thompson, and S. R. Jenkins. 2008. Predator diversity and ecosystem functioning: density modifies the effect of resource partitioning. Ecology 89:298-305.

Haddad, N. M., G. M. Crutsinger, K. Gross, J. Haarstad, and D. Tilman. 2011. Plant diversity and the stability of foodwebs. Ecology Letters 14:42-46.

Heemsbergen, D. A., M. P. Berg, M. Loreau, J. R. van Hal, J. H. Faber, and H. A. Verhoef. 2004. Biodiversity effects on soil processes explained by interspecific functional dissimilarity. Science 306:1019-1020.

Hillebrand, H., and B. Matthiessen. 2009. Biodiversity in a complex world: consolidation and progress in functional biodiversity research. Ecology Letters 12:1405-1419.

Ives, A. R., B. J. Cardinale, and W. E. Snyder. 2005. A synthesis of subdisciplines: predator-prey interactions, and biodiversity and ecosystem functioning. Ecology Letters 8: $102-116$

Ives, A. R., and S. R. Carpenter. 2007. Stability and diversity of ecosystems. Science 317:58-62.

Jessup, C. M., R. Kassen, S. E. Forde, B. Kerr, A. Buckling, P. B. Rainey, and B. J. M. Bohannan. 2004. Big question, small worlds: microbial model system in ecology. Trends in Ecology \& Evolution 19:189-197.

Kokkoris, G. D., V. A. A. Jansen, M. Loreau, and A. Y. Troumbis. 2002. Variability in interaction strength and implications for biodiversity. Journal of Animal Ecology 71:362-371.

Levine, J. M., and J. HilleRisLambers. 2009. The importance of niches for the maintenance of species diversity. Nature 461: $254-257$.

Loreau, M. 1998. Separating sampling and other effects in biodiversity experiments.

Loreau, M., and C. de Mazancourt. 2013. Biodiversity and ecosystem stability: a synthesis of underlying mechanisms. Ecology Letters 16:106-115.

Loreau, M., and A. Hector. 2001. Partitioning selection and complementarity in biodiversity experiments. Nature 412:7276.

Loreau, M., et al. 2001. Ecology-biodiversity and ecosystem functioning: current knowledge and future challenges. Science 294:804-808.

MacArthur, R., and R. Levins. 1967. The limiting similarity, convergence, and divergence of coexisting species. American Naturalist 101:377-385. 
Mächler, E., and F. Altermatt. 2012. Interaction of species traits and environmental disturbance predicts invasion success of aquatic microorganisms. PLoS ONE 7:e45400.

May, R. M. 1972. Will a large complex system be stable? Nature 238:413-415.

McCann, K. S. 2000. The diversity-stability debate. Nature 405:228-233.

McGrady-Steed, J., P. M. Harris, and P. J. Morin. 1997. Biodiversity regulates ecosystem predictability. Nature 390 : 162-165.

Peay, K. G., M. Belisle, and T. Fukami. 2012. Phylogenetic relatedness predicts priority effects in nectar yeast communities. Proceedings of the Royal Society B 279:749-758.

Petchey, O. L., and K. J. Gaston. 2002. Functional diversity (FD), species richness and community composition. Ecology Letters 5:402-411.

Ruesink, J. L., and D. S. Srivastava. 2001. Numerical and per capita responses to species loss: mechanisms maintaining ecosystem function in a community of stream insect detritivores. Oikos 93:221-234.

Saleem, M., I. Fetzer, C. F. Dormann, H. Harms, and A. Chatzinotas. 2012. Predator richness increases the effect of prey diversity on prey yield. Nature Communications 3:1305.

Solè, R. V., and J. M. Montoya. 2001. Complexity and fragility in ecological networks. Proceedings of the Royal Society B 268:2039-2045.
Stouffer, D. B., and J. Bascompte. 2011. Compartmentalization increases food-web persistence. Proceedings of the National Academy of Sciences USA 108:3648-3652.

Suweis, S., F. Simini, J. Banavar, and A. Maritan. 2013. Emergence of structural and dynamical properties of ecological mutualistic networks. Nature 500:449-452.

Tan, J., Z. Pu, A. R. Wade, and L. Jiang. 2012. Species phylogenetic relatedness, priority effects, and ecosystem functioning. Ecology 93:1164-1172.

Tilman, D., C. L. Lehman, and C. E. Bristow. 1998. Diversitystability relationships: Statistical inevitability or ecological consequence? American Naturalist 151:277-282.

Tilman, D., P. B. Reich, J. Knops, D. Wedin, T. Mielke, and C. Lehman. 2001. Diversity and productivity in a long-term grassland experiment. Science 294:843-845.

Turnbull, L. A., J. M. Levine, M. Loreau, and A. Hector. 2013. Coexistence, niches and biodiversity effects on ecosystem functioning. Ecology Letters 16:116-127.

Vandermeer, J. H. 1969. The competitive structure of communities: an experimental approach with protozoa. Ecology 50 : $362-371$.

Violle, C., D. R. Nemergut, Z. Pu, and L. Jiang. 2011. Phylogenetic limiting similarity and competitive exclusion. Ecology Letters 14:782-787.

White, E. P., S. K., M. Ernest, A. J. Kerkhoff, and B. J. Enquist. 2007. Relationships between body size and abundance in ecology. Trends in Ecology \& Evolution 22:323-330.

\section{Supplemental Material}

\section{Ecological Archives}

Appendices A-E are available online: http://dx.doi.org/10.1890/14-1324.1.sm 